For re-submission to CNS Spectrums as an Original Research Article

\title{
Obesity and dissociable forms of impulsivity in young adults
}

\author{
Samuel R Chamberlain MB/BChir PhD MRCPsych ${ }^{1}$, Katherine L Derbyshire ${ }^{2}$, Eric Leppink ${ }^{2}$, \\ Jon E Grant $\mathrm{MD}^{2}$ \\ ${ }^{1}$ Department of Psychiatry \& MRC / Wellcome Trust Behavioural and Clinical Neurosciences \\ Institute, University of Cambridge, Cambridge, United Kingdom; \& Cambridge and \\ Peterborough NHS Foundation Trust (CPFT), United Kingdom \\ ${ }^{2}$ Department of Psychiatry \& Behavioral Neuroscience, University of Chicago, Chicago IL USA
}

Abstract: 248; Body text: 3764; References: 42

\section{Corresponding Author:}

Jon E. Grant, JD, MD, MPH

Professor, Department of Psychiatry \& Behavioral Neuroscience

University of Chicago, Pritzker School of Medicine

5841 S. Maryland Avenue, MC 3077, Chicago, IL 60637

Phone: 773-834-1325; Fax: 773-834-6761

Email: jongrant@uchicago.edu 


\section{Acknowledgements}

This research was supported by a Center for Excellence in Gambling Research grant to Dr. Grant from the National Center for Responsible Gaming (USA); and by the Academy of Medical Sciences (UK; grant to Dr. Chamberlain). Dr. Grant has received research grants from NIDA, the National Center for Responsible Gaming, Psyadon, Transcept, Roche, and Forest Pharmaceuticals, and the University of South Florida. Dr. Grant receives compensation from Springer as the editor-in-chief of the Journal of Gambling Studies and has received royalties from McGraw Hill, Oxford University Press, Norton, and the APPI. Dr. Chamberlain consults for Cambridge Cognition. Ms. Derbyshire and Mr. Leppink report no financial relationships with commercial interest. 


\section{Abstract}

Objective: Obesity is one of the leading causes of preventable morbidity and mortality, and young people are increasingly affected. The aim of this study was to examine relationships between obesity and dissociable forms of impulsivity in young adults. Methods: 511 young adults were recruited from city areas in the United States using media advertisements, and undertook careful and extensive clinical and neurocognitive assessment in order to quantify different aspects of impulsivity (behavioural/phenomenological-, cognitive-, and personalityrelated measures). Associations between obesity and impulsivity were explored using multivariate analysis of variance and discriminant function analysis. $\underline{\text { Results: }} 10.8 \%$ of the sample was obese and $21.5 \%$ was overweight. Compared to controls, subjects with obesity showed significantly elevated rates of maladaptive gambling behaviors, monetary amounts lost to gambling, nicotine consumption, impulsive action (prolonged stop-signal reaction times, StopSignal Test), and impulsive decision-making (reduced modulation of behavior as a function of risk, Cambridge Gamble Test). Even accounting for potential confounding variables, obesity was significantly predicted by female gender, older age, more maladaptive gambling behaviors, and worse inhibitory control (stop-signal reaction times). Conclusion: Obesity is associated with several dissociable forms of impulsivity in young people, especially gambling and impulse dyscontrol. Family doctors should screen for gambling problems in obese young adults. Successful treatment of nicotine dependence in young obese people is likely to require intensive weight management support. Neuropsychological deficits relating to impulsivity occur in obese 
people in early adulthood, and may represent vulnerability markers rather than being due to chronic untoward metabolic effects on brain function.

Keywords: cognition; impulsivity; compulsivity; obesity; overweight; addiction. 


\section{Introduction}

Obesity represents a leading public health concern, and more than 1.4 billion individuals are obese worldwide. Rates of obesity have more than doubled since the 1980s, and epidemiological studies indicate that approximately $30 \%$ of the adult population in the USA are overweight (body mass index, BMI, $\geq 25)$ and that a further $30 \%$ are obese $(\mathrm{BMI} \geq 30)^{1}$. The deleterious long-term consequences of obesity include early death, medical morbidity, and substantial functional impairment. For example, chronic obesity is associated with elevated risk of cardiovascular disease, diabetes, musculoskeletal problems (e.g. arthritis), and some cancers ${ }^{2}$. Multiple tiers of research indicate that interplay between brain function, metabolism, and the external environment are central to our understanding of the development of obesity and its persistence over time ${ }^{3}$.

Despite many calls for action for research into health in young people, this remains a relatively neglected area ${ }^{4}$. Rates of obesity are escalating to a disproportionate extent in young people, who will be exposed to greater cumulative risk over time compared to people developing obesity in later life $e^{5,6}$. Cardiovascular complications and mortality are positively associated with duration of obesity ${ }^{7}$, and obesity in young people leads to subsequent psychiatric complications $^{8}$. Therefore, obesity in young adults represents a key target for the development of early interventions to help reverse excess weight and mitigate unwanted long-term health outcomes. It also represents a useful model or heuristic for studying the pathogenesis of obesity and implicated brain mechanisms, since any identified associations are unlikely to be a consequence of chronic malnutrition or cardiovascular compromise.

The neurobiological underpinnings of obesity can be considered from the perspective of impulsivity ${ }^{3,9-14}$, which can be broadly defined as a tendency towards repetitive or habitual 
behaviors that are premature, risky, or poorly thought out, and which lead to negative longer term outcomes. People with obesity show lack of control over habitual patterns of behavior, and obesity occurs at elevated rates in formal eating disorders such as Binge-Eating Disorder ${ }^{15,16}$, and in Gambling Disorder ${ }^{17}$. Clearly impulsivity is a multifaceted construct, which can be considered from both a clinical (phenomenological) and neurocognitive perspective. Neurobiological models of obesity emphasize the likely involvement of neural circuitry involved in reward and impulsivity, including the mesolimbic system and opioid systems ${ }^{16,18,19}$.

Clinical/phenomenological manifestations of impulsivity can include excess consumption of centrally active substances, presence of impulse control disorders (e.g. Binge-Eating Disorder) and obsessive-compulsive related disorders; and higher than expected scores on questionnairebased measures of impulsivity, such as derived from the Barratt Impulsivity Questionnaire ${ }^{20}$. From a cognitive point of view, impulsivity can be captured in various forms. Motor impulsivity is typically measured using go/no-go and stop-signal paradigms in which volunteers attempt to suppress simple motor responses in response to changing environmental cues. Decision-making impulsivity can be quantified via gambling tasks.

While obesity has been related to 'addiction' conceptually ${ }^{3,14,21,22}$, evidence supporting an epidemiological overlap between obesity and drug/alcohol misuse is mixed, according to a recent review on the topic ${ }^{23}$. However, this review highlighted that much data from site-based studies had been collected from bariatric surgical populations, and that often substance abuse versus dependence had not been delineated. Findings from large-scale epidemiological surveys did not generally identify a significant relationship between obesity and substance misuse ${ }^{23}$. Evidence supporting a relationship between obesity and other manifestations of impulsivity, notably questionnaire- and cognitive-based measures, is stronger. For example, in a recent meta-analysis 
focusing on studies in children, a significant positive relationship between obesity and impulsivity was found overall, when pooling neurocognitive and self-report measures of impulsivity ${ }^{9}$; the relationship remained significant only for the former (neurocognitive measures) when each type was considered separately. Surprisingly few studies exploring the relationship between obesity and cognitive measures exist in adults. In one such study, obesity was associated with impaired performance on a gambling task (preference for larger immediate gain but larger future losses) ${ }^{24}$; elsewhere, impaired response inhibition was identified in women with obesity compared to controls, particularly on latter parts of the cognitive task that was used ${ }^{25}$. Several studies have reported a relationship between impaired decision-making and obesity, particularly a tendency towards more immediate rewards at the expense of larger delayed rewards (referred to as 'temporal discounting') ${ }^{26}$. While individual obesity studies have explored different facets of impulsivity, such as those outlined above, few have explored all of these dissociable aspects of impulsivity in the context of a single study.

Given the considerable public importance of obesity in young people, and limited available data, we explored dissociable clinical and cognitive forms of impulsivity using wellvalidated instruments in a large sample of non-treatment seeking individuals. We hypothesized that obesity would be significantly associated with behavioral manifestations of impulsivity (elevated rates of substance use, rates of gambling, and formal impulse control disorders), neurocognitive forms of impulsivity (impaired response inhibition and preference for risky choices during decision-making), and questionnaire-based measures of impulsivity (Barratt scores).

\section{Method}




\section{Study Participants}

Participants comprised 511 adults aged 18-29 years, recruited consecutively via media advertisements (newspaper and internet advertisements as well as fliers) in the Minneapolis/St Paul and Chicago areas, as part of a broader longitudinal study focusing on impulsivity in young adults. The only inclusion criterion was having gambled in any capacity at least five times in the past 12 months (i.e. a proxy for a minimal level of impulsive behavior).Exclusionary criteria were an inability to provide informed consent, and an inability to understand task procedures. The study was approved by the university institutional review board, and study procedures were carried out in accordance with the Declaration of Helsinki. Due to telephone screening for the above inclusion/exclusion criteria, no one who came for the full in person evaluation and signed the consent was excluded.

\section{Assessments}

After providing written informed consent, participants undertook a detailed interview incorporating clinical and cognitive evaluation, in addition to measurement of height and weight. Occurrence of psychiatric conditions was evaluated using the Mini International Neuropsychiatric Inventory (MINI) ${ }^{27}$, and the Minnesota Impulsive Disorders Interview $(\mathrm{MIDI})^{28}$. The former examines for occurrence of core psychiatric conditions (e.g. depression), while the latter is tailored for detection of impulse control disorders. Quantitative details regarding substance use was collected along with information on gambling behaviors; for the latter, the average amount of dollars lost to gambling per week over the past year was recorded, as were total scores on the Structured Clinical Interview for Pathological Gambling (SCI-PG) ${ }^{29}$. 
The Barratt Impulsivity Scale (BIS) was completed to measure personality-related aspects of impulsivity $^{20}$.

Cognitive testing was completed using a touch-screen computer in a quiet testing environment, supervised by trained test administrators, and included the following tests from the Cambridge Neuropsychological Test Automated Battery (CANTABeclipse, version 3, Cambridge Cognition, UK):

\section{Tests focusing on impulsivity}

Stop-Signal Task (SST). This test measured the ability of subjects to suppress motor responses, i.e. motor impulsivity. A series of directional arrows were presented on the computer screen one per time, and volunteers made rapid motor responses depending on the direction of each arrow (left button for a left arrow and vice versa). On a subset of trials, an auditory 'stop' signal (beep) occurred after presentation of the arrow, and volunteers attempted to suppress their response for the given trial. By varying the time between presentation of the arrow and the stopsignal dynamically, the task calculated a measure of the time taken by the subject to suppress a response that would normally be made, referred to as the stop-signal reaction time (SSRT). Longer SSRTs equated to greater motor impulsivity.

Cambridge Gamble Task (CGT). This test explored various aspects of impulsive decision-making. On each trial, ten boxes were shown on the computer screen, with a token having been hidden behind one of these by the computer. A proportion of these boxes are blue and the rest were red, with the proportions of red to blue boxes being varied pseudo-randomly across trials. Volunteers firstly chose the color they believed the token was hidden behind (red or blue). They then chose what proportion of their points they wished to gamble that they had 
chosen the correct color. Over the course of the task, the aim was to acquire as many points as possible. Key outcome measures were: the overall proportion of points gambled on the task; the overall proportion of rational decisions made (i.e. choice of the logically correct color); and risk adjustment (the extent to which subjects modulate the amount gambled depending on the probability of making correct choices).

Tests focusing on other domains besides impulsivity (control tests)

Intra-dimensional/Extra-dimensional Set-shift (IDED) Task. The IDED examined different aspects of rule learning and cognitive flexibility. Participants viewed two stimuli onscreen on each trial, each comprising two stimulus dimensions (white lines and pink blobs). Through trial and error, participants attempted to learn an underlying rule about which picture was correct based on feedback provided by the computer after each choice (the word 'correct' or 'incorrect' presented on the monitor). After subjects had learnt a given rule, the computer changed it. The primary outcome measure on the test was the total number of errors made, adjusted for any stages of the task that were failed (total errors, adjusted). If this composite measure differs significantly between study groups of interest, the task can be decomposed into different stages to examine the nature of the cognitive learning / cognitive flexibility impairment.

One-Touch Stockings of Cambridge (OTS) Task. This is based on the Tower of Hanoi test of planning ability. Subjects viewed two sets of snooker balls in pockets on each trial, and attempted to work out in their minds the minimum possible number of moves that would be required to make one set of balls match the appearance of the other set. Having thought through each problem, volunteers pressed a numerical button on-screen to indicate what they believed the 
minimum possible number of moves to the correct solution was. The key outcome measure was the number of problems solved correctly on the first attempt.

\section{Data analysis}

Subjects were categorized a priori as being obese $(\mathrm{BMI} \geq 30)$ or overweight $(\mathrm{BMI} \geq 25)$; the remainder of participants served as controls $(\mathrm{BMI}<25)$. Severely underweight individuals $(\mathrm{BMI}<16)$ were excluded (in actuality, this applied to no participants). Primary analysis was a comparison of demographic, clinical, and cognitive parameters between groups using multivariate analysis of variance (MANOVA). The variables included in the analysis were selected due to their potential relevance to obesity, following our review of the existing literature. We were interested in salient demographic characteristics (age, gender, education level, and presence of one or more axis-I psychiatric disorders), clinical features (with an emphasis of substance use, gambling, and impulse control disorders, and questionnaire based scores of impulsivity), and cognitive measures (see above). We first considered whether the MANOVA model was significant overall (multivariate test at the level of group). Then, if the global test was significant, we explored individual variables with Bonferroni correction for multiple comparisons ( $\mathrm{p}<0.05$ corrected) at the level of each domain considered (demographic, behavioral measures of impulsivity, questionnaire measures of impulsivity, neurocognitive measures of impulsivity, and neurocognitive control measures). Individual variables not suited to parametric analysis were explored using alternative statistical methods as indicated in the text, such as chi-square for categorical variables. Where the group level statistic was significant, individual groups were compared using Tukey's least significant difference (LSD) tests. Secondary analysis was discriminant function analysis (step-wise) to identify the combination of 
variables capable of most accurately predicting obese group membership versus control group membership.

\section{Results}

An overview of the demographic, clinical, and cognitive findings is presented in Table 1. The mean (SD) age of the whole sample was 22.0 (3.5) years. The mean (SD) BMI for each respective group was $21.6(2.0), 26.9$ (1.4), and $35.6(5.8)$. The obese individuals were more likely to be female, and smoke and gamble more than the overweight or healthy weight individuals. Alcohol consumption did not differ between groups.

In terms of cognitive issues, obese individuals scored higher on tasks of impulsive action (prolonged stop-signal reaction times, Stop-Signal Test) and impulsive decision-making (reduced modulation of behavior as a function of risk, Cambridge Gamble Test). Overweight subjects did not differ significantly from normal weight individuals on these measures. Both overweight and obese participants showed higher motor impulsivity subscale of the BIS.

Discriminant function analysis identified a model capable of significantly predicting obese versus normal weight status using baseline variables (Wilks' lambda $=0.873$, chisquare $=53.741, \mathrm{p}<0.001)$. The model contained four significant variables (all $\mathrm{p}<0.001)$ : gender, age, SCI-PG scores, and SSRT (standardized canonical discriminant function coefficients of $0.402,+0.674,+0.298$, and +0.307 respectively; note, male gender was positively coded). Of control subjects, $72.0 \%$ were correctly categorized by the model; and for obese subjects, $70.9 \%$ were correctly categorized.

[TABLE 1 AROUND HERE PLEASE] 


\section{Discussion}

This study characterized different forms of impulsivity in obese and overweight young adults, as compared to controls. The proportions of overweight and obese people in our sample were $21.5 \%$ and $10.8 \%$ respectively, rates somewhat lower than observed in Nationwide epidemiological studies in the United States ${ }^{5}$. This may reflect the non-treatment seeking nature of the sample and that our recruits were selected from relatively large cities. The key finding of this study was that obesity was associated with significantly elevated rates of several forms of impulsivity: phenonemological (rates of maladaptive gambling behaviors, nicotine use), neurocognitive (impulsive action and reduced risk adjustment on a decision-making task), and questionnaire-based (motor impulsivity). It was also found that obese group membership (as compared to control group membership) was predicted by female gender, greater age, greater maladaptive gambling behaviors, and worse inhibitory control (impulsive action).

In terms of substance use, obese individuals consumed significantly more nicotine (approximately two-fold higher equivalent cigarette packs per day) than both overweight individuals and controls. Obesity and smoking represent two of the leading causes of preventable deaths in the developed world, and common neurobiological mechanisms have been implicated ${ }^{30}$. Over-indulgence in food and substance addiction are both believed to involve the mesolimbic reward dopamine system, although it is disputed whether this means that obesity should be conceptualized as a 'food addiction' per $s e^{3}$. Overweight individuals may use smoking as a means of trying to regulate their weight, since nicotine can dampen appetite; it is also established that acute increases in weight are frequently observed following smoking cessation ${ }^{31}$. The relationship between smoking and obesity is likely to be complex: cross-sectional studies 
have found that smoking in middle-aged adults is associated with lower BMIs, while - in smokers - greater nicotine consumption correlates positively with $\mathrm{BMI}^{32}$. Our finding of elevated nicotine consumption in young obese adults may indicate that such individuals attempt to regulate their weight by smoking, but that consequent reductions in weight may arise only with longer-term nicotine consumption. Contrary to our expectations, alcohol consumption was no different between the groups. However, the corollary of this is that obesity was unlikely to have been mediated by excess alcohol-related (as opposed to food-related) calorific intake. Furthermore, the lack of elevated use of alcohol in our obese sample would be consistent with the wider literature in that the relationship between obesity and substance misuse/dependence is far from clearly demonstrated ${ }^{23}$.

As hypothesized, obese individuals showed higher rates of maladaptive gambling behaviors (SCI-PG scores) than controls, and larger amounts of money lost to gambling on average per week. While there are limited data exploring rates of maladaptive gambling in obese people, it is well established that individuals with maladaptive gambling behaviors (problem and pathological gambling) show disproportionately elevated rates of obesity, in addition to nicotine use $^{17}$. Prior work suggested that this association could reflect chronic sedentary lifestyles associated with gambling; however, the results here indicate that even young people with obesity show higher occurrence of maladaptive gambling and money lost to gambling.

While the overall rates of psychiatric disorders were numerically higher in obese individuals versus controls, these differences did not obtain statistical significance when corrected for multiple comparisons. Without statistical correction for multiple comparisons, the rate of impulse control disorders would have been significantly higher in obese subjects versus controls, suggesting that the relationship between obesity and these conditions exists, but may 
not be of particularly large effect size. To the knowledge of the authors, no prior studies have quantified impulse control disorders in non-treatment seeking young adults with obesity. In older obese treatment-seeking individuals, $19 \%$ showed one or more impulse control disorders; however, this study lacked a control arm and utilized a different diagnostic instrument ${ }^{33}$. Perhaps the most striking findings in relation to obesity and impulsivity in the current study were those relating to neurocognitive assessment and the questionnaire-based measures, findings redolent of a recent meta-analysis albeit conducted in childhood studies ${ }^{9}$. Groups differed significantly in terms of stop-signal response inhibition, and risk adjustment during the decision-making task. This was due to significantly elevated impulsivity (longer stop-signal reaction times, and less risk adjustment) in obese individuals in comparison to controls. Overweight subjects did not differ significantly from controls on these two cognitive measures. The impulsivity related cognitive deficits in obese participants did not appear to be attributable to more generalized cognitive problems, since this group was not impaired on the control measures. Interestingly, groups also differed on motor impulsivity as measured by the Barratt impulsivity subscale, such that both obese individuals and overweight individuals showed elevated scores versus the controls.

Consistent with our results, previous research found that women with obesity showed impaired stop-signal inhibitory control on latter stages of the task compared to controls ${ }^{25}$; and that obese adolescents showed impaired stop-signal response inhibition the extent of which was predictive of subsequent weight loss via treatment ${ }^{34}$. Furthermore, similar outcomes were found in a smaller study using a modified mental flexibility task that included a pronounced inhibitory control component ${ }^{35}$, and in a study using the Stroop test ${ }^{36}$. Our findings confirm an association between obesity and impaired inhibitory control in adults, a finding that is also consistent with 
meta-analysis of similar studies conducted in children, adolescents, and young adults ${ }^{9}$. Stopsignal inhibitory control is dependent on distributed circuitry including the subthalamic nucleus, inferior frontal cortices, and anterior cingulate cortices. Indeed, using a stop-signal task adapted for functional magnetic resonance imaging (fMRI), women with obesity showed hypoactivation of distributed neural regions involved in inhibitory control (left inferior parietal cortex, supplementary motor area, bilateral insula, and bilateral cuneus) during stop trials, as compared to healthy controls ${ }^{37}$.

To our knowledge, Cambridge Gamble Task (CGT) performance has not previously been examined in relation to obesity. At least five previous studies in adults have measured Iowa Gambling Task (IGT) performance in relation to obesity ${ }^{36}$, and overall these were consistent with obesity being associated with decision-making impairments ${ }^{38}$. The CGT permits fractionation of different aspects of decision-making whereas the IGT is more contingent on trial-by-trial learning. The present findings indicate that decision-making impairments in obesity appear to be selective: obese people failed to modulate their behavior appropriately as a function of risk, but they did not gamble more points, or make irrational choices per se. Decision-making performance on the Cambridge Gamble Task is sensitive to ventromedial prefrontal and insular cortex damage ${ }^{39}$.

The present study, in itself, does not enable conclusions as to the temporal relationship between Stop-signal and CGT impairment and obesity. Animal research suggests that excessive calorific consumption can lead, over time, to structural and functional brain changes (e.g. gliosis, reductions in brain volume, proinflammatory mediators) and consequent cognitive impairment ${ }^{40}$. Initial human neuroimaging studies have identified neural abnormalities such as reduced grey matter density in neural regions including the inferior frontal gyrus, post-central gyrus, and 
putamen, in obesity ${ }^{41}$. We would hypothesize that these cognitive deficits are unlikely to be due to brain changes due to excess calorific consumption, given that we studied young adults (and that similar deficits have been reported in children elsewhere ${ }^{9}$ ), and that the deficits were highly selective rather than global; rather, these deficits may represent predisposing or vulnerability factors, which may also contribute to the persistence of obesity over time. While caution is warranted when inferring the status of neural regions or circuits based on neurocognitive measures alone, the neuropsychological profile of obesity seen here bears remarkable parallels with problem/pathological gambling. When viewed alongside the finding of elevated gambling symptoms in the obese subjects, it is tempting to presuppose a neurobiological relationship between the two.

Some limitations should be considered with respect to the current study. We did not measure historical weight status (for example, for obese individuals, the length of time they had been obese for); however, had we attempted to do so, such measures may well have been inaccurate due to recall bias and subjectivity. Future work should ideally use a longitudinal design to refine our understanding of causal relationships between obesity and impulsivity. This being a naturalistic sample, groups did differ with respect to certain demographic and clinical characteristics - such as the obese group being older and less educated, with a greater proportion of females. As groups were not 'pre-matched' for these characteristics, these variables represent potential confounds, which should be evaluated further in future larger studies. That said, gender, age, SCI-PG scores, and SSRT, all independently predicted obesity: therefore even accounting 
for other variables, the relationship between obesity and two forms of impulsivity (gambling behavior and impulse dyscontrol) remained robust.

\section{Conclusion}

In summary, obesity in young adults was associated with higher rates of various manifestations of impulsivity as compared to controls: these took the form of behaviors (higher rates of gambling and nicotine consumption), objective cognitive measures (stop-signal and decision-making deficits), and a questionnaire-based subscore (Barratt motor impulsiveness). However, the strongest relationships with obesity were for gambling symptoms and impulse dyscontrol, which were robust even accounting for potential confounding variables (e.g. age, education).

These findings have a number of clinical and scientific implications. Family doctors should be careful to screen for gambling problems in obese people, to identify unmet treatment needs. The data also raise questions regarding the relationship between smoking and obesity they demonstrate that young adults with obesity smoke twice as much as normal weight individuals. Given that smoking cessation can lead to weight gain, treating nicotine dependence is unlikely to be successful without mentoring and concomitant weight loss programs. The study shows that cognitive problems relating to impulsivity occur early in the pathophysiology of obesity, raising the prospect that they may represent candidate vulnerability markers (rather than a consequence of obesity). The finding of cognitive deficits in obese subjects akin to those seen elsewhere in pathological gambling, alongside the elevated rates of gambling behavior in the obese subjects, is suggestive of an aetiological relationship between these two types of behavior. Confirmation of the precise relationship between obesity, gambling, impulse dyscontrol, and 
impaired decision-making, requires further research to elucidate. Future work should explore

these associations longitudinally and their functional impact, and evaluate the neurobiological substrates of the cognitive impairments, including relationships with genetic polymorphisms involved in cognition ${ }^{42}$.

\section{References}

1. Flegal KM, Carroll MD, Ogden CL, Curtin LR. Prevalence and trends in obesity among US adults, 1999-2008. Jama. Jan 20 2010;303(3):235-241.

2. Kelsey MM, Zaepfel A, Bjornstad P, Nadeau KJ. Age-Related Consequences of Childhood Obesity. Gerontology. Jan 92014.

3. Ziauddeen $\mathrm{H}$, Fletcher PC. Is food addiction a valid and useful concept? Obesity reviews : an official journal of the International Association for the Study of Obesity. Jan 2013;14(1):19-28.

4. Patton GC, Ross DA, Santelli JS, Sawyer SM, Viner RM, Kleinert S. Next steps for adolescent health: a Lancet Commission. Lancet. Feb 1 2014;383(9915):385-386.

5. Flegal KM, Carroll MD, Kit BK, Ogden CL. Prevalence of obesity and trends in the distribution of body mass index among US adults, 1999-2010. Jama. Feb 1 2012;307(5):491-497.

6. Racette SB, Deusinger SS, Strube MJ, Highstein GR, Deusinger RH. Weight changes, exercise, and dietary patterns during freshman and sophomore years of college. Journal of American college health : $J$ of ACH. May-Jun 2005;53(6):245-251.

7. Reis JP, Hankinson AL, Loria CM, et al. Duration of abdominal obesity beginning in young adulthood and incident diabetes through middle age: the CARDIA study. Diabetes care. May 2013;36(5):1241-1247.

8. Sanchez-Villegas A, Field AE, O'Reilly EJ, et al. Perceived and actual obesity in childhood and adolescence and risk of adult depression. Journal of epidemiology and community health. Jan 2013;67(1):81-86.

9. Thamotharan S, Lange K, Zale EL, Huffhines L, Fields S. The role of impulsivity in pediatric obesity and weight status: a meta-analytic review. Clinical psychology review. Mar 2013;33(2):253-262.

10. Jasinska AJ, Yasuda M, Burant CF, et al. Impulsivity and inhibitory control deficits are associated with unhealthy eating in young adults. Appetite. Dec 2012;59(3):738-747.

11. Schag K, Schonleber J, Teufel M, Zipfel S, Giel KE. Food-related impulsivity in obesity and binge eating disorder--a systematic review. Obesity reviews : an official journal of the International Association for the Study of Obesity. Jun 2013;14(6):477-495.

12. Moreno C, Tandon R. Should overeating and obesity be classified as an addictive disorder in DSM-5? Current pharmaceutical design. 2011;17(12):1128-1131.

13. Markus A. Neurobiology of obesity. Nature neuroscience. May 2005;8(5):551.

14. Blumenthal DM, Gold MS. Neurobiology of food addiction. Current opinion in clinical nutrition and metabolic care. Jul 2010;13(4):359-365. 
15. Villarejo C, Fernandez-Aranda F, Jimenez-Murcia S, et al. Lifetime obesity in patients with eating disorders: increasing prevalence, clinical and personality correlates. European eating disorders review : the journal of the Eating Disorders Association. May 2012;20(3):250-254.

16. Favaro A. Brain development and neurocircuit modeling are the interface between genetic/environmental risk factors and eating disorders. A commentary on Keel \& Forney and Friederich et al. The International journal of eating disorders. Jul 2013;46(5):443446.

17. Black DW, Shaw M, McCormick B, Allen J. Pathological gambling: relationship to obesity, self-reported chronic medical conditions, poor lifestyle choices, and impaired quality of life. Comprehensive psychiatry. Feb 2013;54(2):97-104.

18. Stice E, Figlewicz DP, Gosnell BA, Levine AS, Pratt WE. The contribution of brain reward circuits to the obesity epidemic. Neurosci Biobehav Rev. Nov 2013;37(9 Pt A):2047-2058.

19. Casper RC, Sullivan EL, Tecott L. Relevance of animal models to human eating disorders and obesity. Psychopharmacology (Berl). Aug 2008;199(3):313-329.

20. Barratt ES. Factor analysis of some psychometric measures of impulsiveness and anxiety,. Psychological reports. 1965;16:547-554.

21. Volkow ND, Wang GJ, Fowler JS, Telang F. Overlapping neuronal circuits in addiction and obesity: evidence of systems pathology. Philosophical transactions of the Royal Society of London. Series B, Biological sciences. Oct 12 2008;363(1507):3191-3200.

22. Dagher A. The neurobiology of appetite: hunger as addiction. International journal of obesity. Jun 2009;33 Suppl 2:S30-33.

23. Sansone RA, Sansone LA. Obesity and substance misuse: is there a relationship? Innovations in clinical neuroscience. Sep 2013;10(9-10):30-35.

24. Davis C, Levitan RD, Muglia P, Bewell C, Kennedy JL. Decision-making deficits and overeating: a risk model for obesity. Obesity research. Jun 2004;12(6):929-935.

25. Nederkoorn C, Smulders FT, Havermans RC, Roefs A, Jansen A. Impulsivity in obese women. Appetite. Sep 2006;47(2):253-256.

26. Epstein LH, Jankowiak N, Fletcher KD, et al. Women who are motivated to eat and discount the future are more obese. Obesity (Silver Spring). Jun 2014;22(6):1394-1399.

27. Sheehan DV, Lecrubier Y, Sheehan KH, et al. The Mini-International Neuropsychiatric Interview (M.I.N.I.): the development and validation of a structured diagnostic psychiatric interview for DSM-IV and ICD-10. The Journal of clinical psychiatry. 1998;59 Suppl 20:22-33;quiz 34-57.

28. Odlaug BL, Grant JE. Impulse-control disorders in a college sample: results from the self-administered Minnesota Impulse Disorders Interview (MIDI). Primary care companion to the Journal of clinical psychiatry. 2010;12(2).

29. Grant JE, Steinberg MA, Kim SW, Rounsaville BJ, Potenza MN. Preliminary validity and reliability testing of a structured clinical interview for pathological gambling. Psychiatry Res. Aug 30 2004;128(1):79-88.

30. Thorgeirsson TE, Gudbjartsson DF, Sulem P, et al. A common biological basis of obesity and nicotine addiction. Translational psychiatry. 2013;3:e308.

31. Audrain-McGovern J, Benowitz NL. Cigarette smoking, nicotine, and body weight. Clin Pharmacol Ther. Jul 2011;90(1):164-168. 
32. Clair C, Chiolero A, Faeh D, et al. Dose-dependent positive association between cigarette smoking, abdominal obesity and body fat: cross-sectional data from a population-based survey. BMC public health. 2011;11:23.

33. Schmidt F, Korber S, de Zwaan M, Muller A. Impulse control disorders in obese patients. European eating disorders review : the journal of the Eating Disorders Association. May 2012;20(3):e144-147.

34. Kulendran M, Vlaev I, Sugden C, et al. Neuropsychological assessment as a predictor of weight loss in obese adolescents. International journal of obesity. Oct 292013.

35. Mobbs O, Iglesias K, Golay A, Van der Linden M. Cognitive deficits in obese persons with and without binge eating disorder. Investigation using a mental flexibility task. Appetite. Aug 2011;57(1):263-271.

36. Fagundo AB, de la Torre R, Jimenez-Murcia S, et al. Executive functions profile in extreme eating/weight conditions: from anorexia nervosa to obesity. PloS one. 2012;7(8):e43382.

37. Hendrick OM, Luo X, Zhang S, Li CS. Saliency processing and obesity: a preliminary imaging study of the stop signal task. Obesity (Silver Spring). Sep 2012;20(9):1796-1802.

38. Fitzpatrick S, Gilbert S, Serpell L. Systematic review: are overweight and obese individuals impaired on behavioural tasks of executive functioning? Neuropsychol Rev. Jun 2013;23(2):138-156.

39. Clark L, Bechara A, Damasio H, Aitken MR, Sahakian BJ, Robbins TW. Differential effects of insular and ventromedial prefrontal cortex lesions on risky decision-making. Brain : a journal of neurology. May 2008;131(Pt 5):1311-1322.

40. Shefer G, Marcus Y, Stern N. Is obesity a brain disease? Neurosci Biobehav Rev. Dec 2013;37(10 Pt 2):2489-2503.

41. Pannacciulli N, Del Parigi A, Chen K, Le DS, Reiman EM, Tataranni PA. Brain abnormalities in human obesity: a voxel-based morphometric study. NeuroImage. Jul 15 2006;31(4):1419-1425.

42. Favaro A, Clementi M, Manara R, et al. Catechol-O-methyltransferase genotype modifies executive functioning and prefrontal functional connectivity in women with anorexia nervosa. Journal of psychiatry \& neuroscience : JPN. Jul 2013;38(4):241-248. 